\title{
Cytomegalovirus pulls strings behind NK cells
}

\section{Norimitsu Kadowaki, Kenichi Ishiyama and Toshio Kitawaki}

Tyrosine kinase inhibitors (TKIs) targeting the BCRABL1 kinase have revolutionized the treatment strategy for Philadelphia chromosome-positive $\left(\mathrm{Ph}^{+}\right)$chronic myelogenous leukemia (CML) and acute lymphoblastic leukemia (ALL). Among the TKIs, dasatinib is distinctive in that it inhibits a broad spectrum of tyrosine kinases, including key regulators of immune responses [1]. In agreement with this profile, dasatinib suppresses activity of various immune cells, such as T cells, NK cells, and plasmacytoid dendritic cells in vitro. Meanwhile, a unique immunological phenomenon, expansion of large granular lymphocytes (LGLs), has been frequently observed in $\mathrm{Ph}^{+}$ leukemia patients treated with dasatinib. Such expansion does not occur with other TKIs, imatinib or nilotinib. Importantly, LGL expansion during dasatinib treatment is associated with superior therapeutic responses [2]. Thus, elucidating the mechanisms underlying the LGL expansion is instrumental in improving the treatment outcome for $\mathrm{Ph}^{+}$ leukemia.

Here is an enigma; dasatinib is immunosuppressive in vitro, whereas it is immunostimulatory in vivo. How can we reconcile these apparently opposite phenomena? A previous study implicated cytomegalovirus (CMV) in the LGL expansion, since it is predominantly observed in CMV-seropositive $\left(\mathrm{CMV}^{+}\right)$patients [3]. However, CMV reactivation is observed only in a small fraction of patients, and therefore, underlying factors in the lymphocytosis were uncertain. Thus, we aimed to identify them.

LGLs are composed of $\mathrm{CD}^{+} \mathrm{T}$ cells, $\gamma \delta \mathrm{T}$ cells, and NK cells, collectively cytotoxic lymphocytes. We first identified NK cells as the dominant LGLs expanding in dasatinib-treated patients. All the patients with NK cell expansion were $\mathrm{CMV}^{+}$. NK cells express a complex mosaic of inhibitory and activating receptors with large variation among individuals. In order to assess the accurate status of such variegated cells, we performed multiparametric phenotyping of NK cells from healthy donors and $\mathrm{Ph}^{+}$leukemia patients treated with imatinib, nilotinib or dasatinib. We then analyzed the data using principal component analysis (PCA), a mathematical algorithm that reduces dimensionality of multiparametric data by defining a novel parameter (principal component) as a combination of the parameters to preserve the most essential characteristics of the dataset. PCA revealed that $\mathrm{NK}$ cells from $\mathrm{CMV}^{+}$dasatinib-treated patients underwent phenotypic progression that reflects $\mathrm{CMV}$ associated differentiation (such as $\mathrm{NKG} 2 \mathrm{C}^{\text {high }} \mathrm{CD} 57^{\text {high }}$
LIR-1 $\left.1^{\text {high }} \mathrm{NKp} 30^{\text {low }} \mathrm{NKp} 46^{\text {low }}\right)$. NK cells from $\mathrm{CMV}^{+}$ imatinib- or nilotinib-treated patients and $\mathrm{CMV}^{+}$healthy donors had an intermediate phenotype, and those from CMV-seronegative individuals did not have the CMVassociated phenotype. Seven of $30 \mathrm{CMV}^{+}$dasatinibtreated patients developed $\mathrm{CMV}$ reactivation as defined by an elevation of CMV-IgM or a positive result of PCR. Notably, the CMV-associated highly differentiated status of NK cells was already observed at leukemia diagnosis in the majority of $\mathrm{CMV}^{+}$patients, and was further enhanced after starting dasatinib in virtually all $\mathrm{CMV}^{+}$patients. CMV reactivation was detected in 8 of $20 \mathrm{CMV}^{+}$patients at leukemia diagnosis. Importantly, the patients with a higher degree of NK cell differentiation at diagnosis underwent significantly greater NK cell expansion and faster decreases in $B C R-A B L 1$ mRNA after starting dasatinib. Thus, pretreatment differentiated status of NK cells predicts robust expansion of NK cells and an earlier clinical response after starting dasatinib in $\mathrm{CMV}^{+}$patients.

In this study, the PCA revealed that virtually all the $\mathrm{CMV}^{+}$patients exhibited enhancement of CMV-associated NK cell differentiation during dasatinib treatment, regardless of the presence or absence of documented CMV reactivation. We assume that a low level of continuous

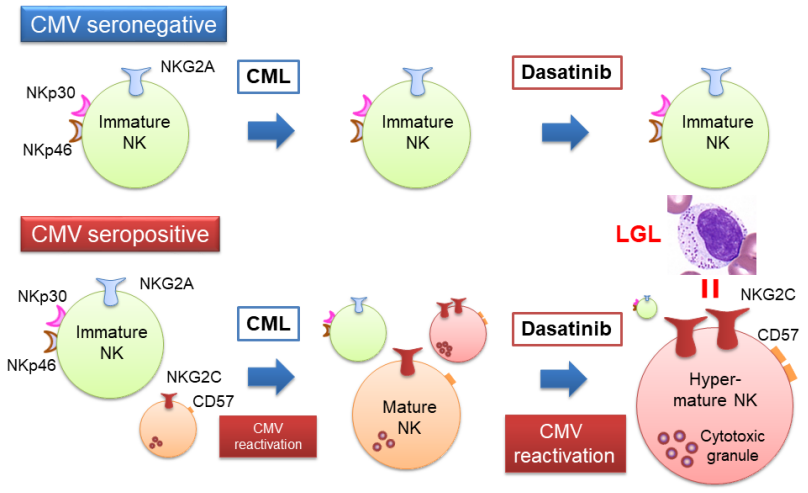

Figure 1: Mechanisms of NK cell expansion in CMVseropositive patients treated with dasatinib. In CMVseronegative patients, CMV-associated NK cells are not observed during the whole course of CML onset and dasatinib treatment, and virtually all the $\mathrm{NK}$ cells are $\mathrm{NKG}_{2} \mathrm{~A}^{+} \mathrm{NKG} 2 \mathrm{C}^{-}$ NKp30 high $N K p 46^{\text {high }}$. In contrast, in CMV-seropositive patients, a small number of $\mathrm{CMV}$-associated $\mathrm{NKG}_{2} \mathrm{C}^{+} \mathrm{CD} 57^{+} \mathrm{NK}$ cells have been generated. The CML onset appears to trigger subclinical CMV reactivation and consequent activation of the CMV-associated NK cells. Dasatinib treatment further reactivates $\mathrm{CMV}$, leading to further activation and proliferation of the NK cells, which are recognized as LGLs. 
CMV reactivation, often below detection levels of assay, is responsible for the NK cell differentiation. Furthermore, similar enhancement of NK cell differentiation was observed with mere presence of leukemia, exclusively again in $\mathrm{CMV}^{+}$patients. Based on these comprehensive analyses, we propose CMV as an initiation factor followed by leukemia and dasatinib as enhancing factors in the NK cell activation (Figure 1).

Notably, the CMV-associated $\mathrm{NKG}_{2} \mathrm{C}^{+} \mathrm{NK}$ cells are considered to represent the human counterpart of mouse memory NK cells, and acquire distinctive molecular signature by epigenetic modification (FcR $\gamma^{-} \mathrm{PLZF}^{-} \mathrm{SYK}$ EAT-2 ${ }^{-}$[4]. It is possible that the prompt expansion of NK cells with higher degrees of differentiation after starting dasatinib may reflect the property of immunological memory. Since such adaptive NK cells also appear to reduce relapse of hematological malignancies in allogeneic transplant patients who experienced CMV reactivation [5], the CMV-associated distinctive type of adaptive NK cells may contribute to suppressing leukemia relapse in transplant patients as well as in dasatinib-treated patients.

An important issue in CML treatment is whether TKIs can be discontinued after achieving a deep molecular response. A substantial proportion of such patients maintains the response for more than 12 months after discontinuation of TKIs. Notably, increases in NK cells [6, 7] but not $T$ cells [7] associate with longer treatment-free remission, implying that immunological surveillance by NK cells is involved in keeping CML under control. Thus, the CMV-associated NK cell activation may contribute to preventing CML relapse after discontinuing dasatinib.

Our study using PCA suggests that the distinctive NK cell subset, that is, CMV-associated adaptive NK cells, is responsible for the dasatinib-induced NK cell expansion, which likely occurs through subclinical CMV reactivation. Intriguingly, the $\mathrm{CMV}$-associated highly differentiated status is already observed at leukemia diagnosis in a large proportion of patients, and predicts prompt expansion of NK cells after starting dasatinib. These findings provide a rationale for the exploitation of CMV-associated $\mathrm{NK}$ cell activation to improve the treatment outcome for $\mathrm{Ph}^{+}$leukemia. Furthermore, the findings may illustrate exploitation of immunity against resident viruses, virobiota, for the control of malignancies [8].
Norimitsu Kadowaki: Department of Internal Medicine, Division of Hematology, Rheumatology and Respiratory Medicine, Faculty of Medicine, Kagawa University, Kagawa, Japan

Correspondence to: Norimitsu Kadowaki, email kado@med. kagawa-u.ac.jp

Keywords: dasatinib, $\mathrm{Ph}^{+}$leukemia, cytomegalovirus, NK cells, principal component analysis

Received: September 07, 2017

Published: September 28, 2017

\section{REFERENCES}

1. Li J, et al. Nat Chem Biol. 2010; 6:291-299.

2. Kim DH, et al. Haematologica. 2009; 94:135-139.

3. Kreutzman A, et al. Leukemia. 2011; 25:1587-1597.

4. Rölle A, et al. Trends Immunol. 2016; 37:233-243.

5. Schlums H, et al. Immunity. 2015; 42:443-456.

6. Mizoguchi I, et al. Cancer Sci. 2013; 104:1146-1153.

7. Imagawa J, et al. Lancet Haematol. 2015; 2:e528-535.

8. Duerkop BA, et al. Nat Immunol. 2013; 14:654-659.

Copyright: Kadowaki et al. This is an open-access article distributed under the terms of the Creative Commons Attribution License 3.0 (CC BY 3.0), which permits unrestricted use, distribution, and reproduction in any medium, provided the original author and source are credited. 